\title{
Long term treatment of pulmonary arterial hypertension with beraprost, an oral prostacyclin analogue
}

C D Vizza, S Sciomer, S Morelli, C Lavalle, P Di Marzio, D Padovani, R Badagliacca, A R Vestri, R Naeije, F Fedele

\begin{abstract}
Objective-To evaluate the effects of one year's treatment with beraprost, an orally active prostacyclin analogue, in patients with severe pulmonary hypertension.

Patients-13 patients with severe pulmonary hypertension. This was primary in nine, thromboembolic in three, and caused by Eisenmenger syndrome in one.

Methods-All patients underwent right heart catheterisation. Mean (SD) right atrial pressure was 5 (3) $\mathrm{mm} \mathrm{Hg}$, mean pulmonary artery pressure was 48 (12) $\mathrm{mm} \mathrm{Hg}$, cardiac index was 2.6 (0.8) $1 / \mathrm{min} / \mathrm{m}^{2}$, and mixed venous oxygen saturation was $68(7) \%$. Beraprost was started at the dose of $20 \mu \mathrm{g}$ three to four times a day $(1 \mu \mathrm{g} / \mathrm{kg} /$ day), increasing after one month to $40 \mu \mathrm{g}$ three to four times a day $(2 \mu \mathrm{g} / \mathrm{kg} /$ day), with further increases of $20 \mu \mathrm{g}$ three to four times a day in case of clinical deterioration.

Main outcome measures-New York Heart Association (NYHA) functional class, exercise capacity measured by distance walked in six minutes, and systolic pulmonary pressure (by echocardiography) were evaluated at baseline, after one month's treatment, and then every three months for a year.

Results-After the first month of treatment, NYHA class decreased from $3.4(0.7)$ to 2.9 (0.7) $(\mathrm{p}<0.05)$, the six minute walking distance increased from $213(64)$ to $276(101) \mathrm{m}(\mathrm{p}<0.05)$, and systolic pulmonary artery pressure decreased from 93 (15) to 85 (18) $\mathrm{mm} \mathrm{Hg}$ (NS). One patient died after 40 days from refractory right heart failure, and another was lost for follow up at six months. The 11 remaining patients had persistent improvements in functional class and exercise capacity and a significant decrease in systolic pulmonary artery pressure in the period from 1-12 months. Side effects were minor.
\end{abstract}

Conclusions-Oral administration of beraprost may result in long lasting clinical and haemodynamic improvements in patients with severe pulmonary hypertension.

(Heart 2001;86:661-665)

Keywords: pulmonary arterial hypertension; prostacyclin; beraprost

Continuous intravenous epoprostenol (prostacyclin) has been shown to improve the functional state, exercise capacity, pulmonary haemodynamics, and survival in patients with primary pulmonary hypertension. ${ }^{1-6}$ Similar clinical effects of intravenous epoprostenol treatment have been reported in patients with pulmonary hypertension secondary to connective tissue disease, ${ }^{78}$ congenital heart defects, ${ }^{9}{ }^{10}$ and peripheral chronic thromboembolism. ${ }^{10}$ However, chronic intravenous epoprostenol requires a permanently implanted central venous catheter and a portable infusion pump, which exposes the patient to a series of complications including catheter related thrombosis, infection, and delivery system malfunction resulting in rapid underdosing or overdosing of a drug with a very short half life. ${ }^{1-10}$ Because of these drawbacks, alternative modes of administration and longer acting prostacyclin derivatives are currently under investigation.

Favourable results have been reported in patients with primary and secondary severe pulmonary hypertension treated with nebulised iloprost. However, the relatively short half life of this prostacyclin analogue imposes the need for up to 12 inhalations a day, each of which lasts up to 15 minutes, ${ }^{11}{ }^{12}$ while the use of aerosol nebulisers is cumbersome. Hopes have therefore been raised by preliminary Japanese studies reporting beneficial effects of the oral prostacyclin analogue beraprost in patients with primary pulmonary hypertension..$^{13-15}$

We here report our experience of 13 patients with severe pulmonary hypertension treated with oral beraprost and followed for one year. Satisfactory clinical improvement was observed in 11 of these patients.

\section{Methods}

STUDY PATIENTS

Thirteen consecutive patients with severe pulmonary hypertension (four male and nine female), aged between 11 and 62 years (mean age 45 years), gave their informed consent to the study, which had been approved by the local ethics committee. They were referred to our centre between January 1998 and January 2000, at a time when intravenous epoprostenol treatment was not yet available in Italy. The patients were diagnosed as having either primary (nine patients), peripheral thromboembolic (three), or congenital heart disease associated (one patient) pulmonary hypertension. Dyspnoea was the main symptom in all patients, while six had chest pain and seven syncope. The duration of symptoms ranged from 1-36 months, mean (SD) 14 (10) months. All the patients had severe 
functional limitation as assessed by the New York Heart Association (NYHA) classification: six were in class IV, six others in class III, and only one in class II.

The diagnosis of primary pulmonary hypertension relied on right heart catheterisation showing a mean pulmonary artery pressure higher than $25 \mathrm{~mm} \mathrm{Hg}$, and the use of an algorithm incorporating a mandatory chest $x$ ray, respiratory function tests, a perfusion lung scan, an echocardiogram, arterial blood gas analysis, and the results of the right heart catheterisation to exclude secondary causes. ${ }^{16}$ The diagnosis of chronic peripheral inoperable thromboembolic pulmonary hypertension was based on significant perfusion abnormalities on a ventilation/perfusion lung scan, and confirmed by a pulmonary angiogram. ${ }^{17}$ The diagnosis of ventricular septal defect was made by echocardiography.

STUDY PROTOCOL

After the diagnostic right heart catheterisation, which included an acute vasodilator challenge with 20-60 ppm inhaled nitric oxide, the patients were managed with conventional treatment including digitalis, diuretics, nitrates, oral anticoagulants, and nifedipine when indicated. ${ }^{18}$ Conventional treatment was titrated in 3-4 weeks in order to obtain the maximum clinical effect.

At the end of this period, the patients had a clinical evaluation, a Doppler echocardiogram, and two, six minute walk tests (on successive days). The best of the two walking tests was used as the baseline result. Beraprost was then added to the standard treatment, starting at a dose of $20 \mu \mathrm{g}$ three to four times a day $(1 \mu \mathrm{g} / \mathrm{kg} /$ day) and progressively increasing to a maximum dose of $2 \mu \mathrm{g} / \mathrm{kg} /$ day, or as tolerated. The patients were followed up with a clinical examination, a six minute walk test, and an echocardiogram at 1, 3, 6, 9, and 12 months. In addition, telephone contact was made at least twice a month.

The dose of beraprost was increased if there was clinical deterioration, in increments of 5-10- $\mu$ g three to four times a day until improvement occurred or until there were adverse effects. If hypotension occurred, the other vasodilators were reduced or stopped before considering a stepwise reduction in the beraprost dose. The dose of diuretics was increased if there was weight gain and clinical signs of right heart failure. During the treatment period, all measurements were performed 3-4 hours after the last dose of beraprost had been taken.

Exercise capacity was measured by an unencouraged six minute walk test performed in a $25 \mathrm{~m}$ length of corridor under the same environmental conditions and at about the same time of day ( \pm 2 hours). ${ }^{19}$ Systolic pulmonary arterial pressure (Ppa) was estimated by flow Doppler echocardiography from the maximum velocity of the tricuspid regurgitant flow. ${ }^{20}$

STATISTICAL ANALYSIS

Data are expressed as mean (SD), except when indicated. A repeated measures analysis of
Table 1 Baseline haemodynamics and six minute walk distance

\begin{tabular}{ll}
\hline Variable & Mean (SD) \\
\hline Distance $(\mathrm{m})$ & $213(64)$ \\
Right atrial pressure (mm $\mathrm{Hg})$ & $5(3)$ \\
Pulmonary artery pressure $(\mathrm{mm} \mathrm{Hg})$ & $48(12)$ \\
Paop $(\mathrm{mm} \mathrm{Hg})$ & $7(4)$ \\
Cardiac index $\left(1 / \mathrm{min} / \mathrm{m}^{2}\right)$ & $2.6(0.8)$ \\
Pulmonary vascular resistance (Wood units) & $14.0(7.6)$ \\
Mixed venous oxygen saturation (\%) & $68(7)$ \\
\hline
\end{tabular}

Paop, pulmonary artery occluded pressure

Table 2 Concomitant treatment

\begin{tabular}{lll}
\hline Drug & $\begin{array}{l}\text { Number of patients } \\
\text { treated }\end{array}$ & Daily dose \\
\hline Digoxin & $13 / 13$ & $0.125-0.25 \mathrm{mg}$ \\
Frusemide & $13 / 13$ & $25-125 \mathrm{mg}$ \\
Warfarin & $11 / 13$ & $4-6 \mathrm{mg}$ \\
Nifedipine & $3 / 13$ & $40-60 \mathrm{mg}$ \\
Enalapril & $2 / 13$ & $5 \mathrm{mg}$ \\
Nitrates & $2 / 13$ & $60-80 \mathrm{mg}$ \\
Amiodarone & $1 / 13$ & $200 \mathrm{mg}$ \\
\hline
\end{tabular}

variance was applied to the series of monthly measurements, with a correction for the missing data in the patient who died after 40 days and the patient who left the study after the six month visit. ${ }^{21}$ Modified $t$ tests (that is, $t$ tests with the residual variance of the analysis of variance) were used to compare follow up measurements with the baseline when the $F$ ratio of the analysis of variance reached a critical value of $\mathrm{p}<0.05 .^{21}$

\section{Results}

The initial values for invasive haemodynamic measurements are shown in table 1. The patients all had severe pulmonary hypertension with a low cardiac output and on average normal right and left heart filling pressures. The right atrial pressure was above $10 \mathrm{~mm} \mathrm{Hg}$ in three patients.

The baseline conventional treatment after the one month run in period is summarised in table 2. Three patients were given nifedipine on the basis of $>30 \%$ reversibility of pulmonary vascular resistance on acute reversibility testing with inhaled nitric oxide. Two patients did not receive anticoagulant treatment because of previous gastrointestinal bleeding. One patient was on prophylactic amiodarone because of recurrent atrial fibrillation. There was no change in the clinical state of the patients during the one month run in period.

The individual daily beraprost doses at one month and 12 months of treatment are shown in table 3 . The mean daily beraprost dose was 116 (24) $\mu \mathrm{g}$ (range 80-160 $\mu \mathrm{g}$ ) after the first month of treatment, and increased to 193 $(74 \mu \mathrm{g})$ (range $100-360 \mu \mathrm{g})$ at 12 months. All the patients needed at least one increase in dose (range 1-5). Angiotensin converting enzyme inhibitors were stopped in two patients, the diuretic regimen was altered by adding aldactone $25 \mathrm{mg} /$ day and reducing frusemide (furosemide) in three patients, and amiodarone was instituted in two patients to treat an episode of atrial fibrillation. At each follow up visit all the patients were in sinus rhythm. 
Table 3 Daily dose of beraprost at 1 and 12 months of treatment

\begin{tabular}{llllll}
\hline Patient & Age (years) & Weight $(\mathrm{kg})$ & $\begin{array}{l}\text { Dose }(\mu \mathrm{g}) \text { after } \\
\text { 1 month }\end{array}$ & $\begin{array}{l}\text { Dose }(\mu \mathrm{g}) \text { after } \\
12 \text { months }\end{array}$ & Increments \\
\hline 1 & 58 & 65 & 120 & 200 & 3 \\
2 & 42 & 52 & 100 & 160 & 2 \\
3 & 58 & 65 & 120 & 160 & 1 \\
4 & 62 & 70 & 80 & 140 & 2 \\
5 & 33 & 58 & 160 & 360 & 5 \\
6 & 56 & 58 & 80 & 100 & 2 \\
7 & 36 & 52 & 120 & 160 & 1 \\
8 & 34 & 80 & 160 & 240 & 2 \\
9 & 11 & 42 & 100 & 280 & 3 \\
10 & 52 & 53 & 120 & 160 & 1 \\
11 & 55 & 57 & 120 & & \\
12 & 45 & 56 & 120 & 160 & 1 \\
13 & 38 & 64 & 120 & & \\
\hline
\end{tabular}

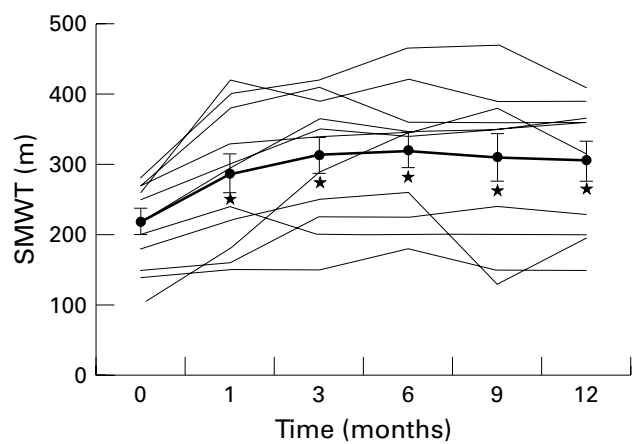

Figure 1 Individual values (thin lines) of distances walked in six minutes (SMWT) in patients with severe pulmonary hypertension at baseline and after 1, 3, 6, 9, and 12 months of treatment with oral beraprost. Thick lines with data points and vertical bars represent the mean responses with SEM. ${ }^{\star} p<0.05 v$ baseline.

One patient died after 40 days from refractory right ventricular failure. One patient moved abroad and was lost to follow up after six months. Follow up for the full 12 months was therefore achieved in 11 patients.

NYHA class improved at one month from $3.4(0.7)$ to $2.9(0.7) \quad(\mathrm{p}<0.016)$, and remained unchanged thereafter. Two patients had no modification in NYHA class, five had a stable improvement by one class, and three had an improvement by two classes. Three patients had fluctuating behaviour with initial improvement followed by deterioration.

The six minute walk distances at baseline and during beraprost treatment are shown in fig 1 . The baseline six minute walk distance was 213 (64) metres. This increased by 63 (47) metres at one month and the improvement was maintained during the subsequent months.

Systolic pulmonary artery pressures at baseline and during beraprost treatment are shown in fig 2. Systolic pulmonary artery pressures decreased. However, the $\mathrm{F}$ value of the analysis of variance showed a borderline $\mathrm{p}$ value of 0.06 . We nevertheless applied modified $t$ tests to compare beraprost treatment with baseline measurements, which showed a decrease in pulmonary artery pressure that was significant from the third to the 12th month $(\mathrm{p}<0.01)$.

The side effects of beraprost consisted of skin flushing $(\mathrm{n}=12)$, rash $(\mathrm{n}=4)$, mild diarrhoea in $(\mathrm{n}=3)$, muscular pain $(\mathrm{n}=2)$, and gastric discomfort $(n=4)$. A temporary reduction in dose because of side effects was necessary only once in two patients. One patient had a muscular haematoma related to anticoagulation (INR $>6$ ).

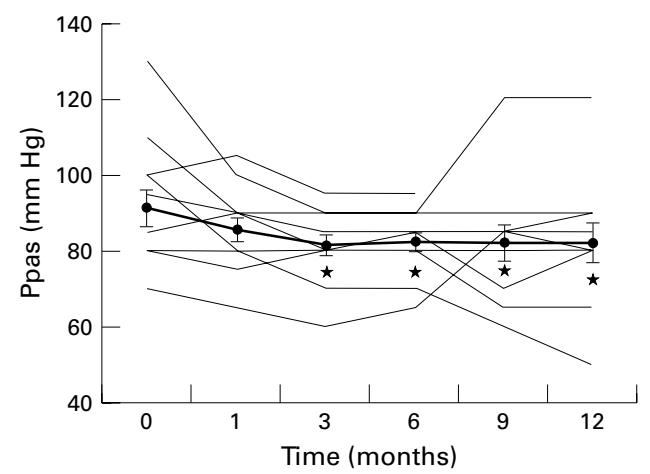

Figure 2 Systolic pulmonary artery pressures (Ppas) estimated by Doppler echocardiography in patients with severe pulmonary hypertension at baseline and after 1, 3, 6 , 9 , and 12 months of treatment with oral beraprost. Thick lines with data points and vertical bars represent the mean responses with SEM. ${ }^{\star} p<0.05 v$ baseline.

\section{Discussion}

This prospective uncontrolled study shows that the oral intake of the prostacyclin analogue beraprost in patients with severe pulmonary hypertension is well tolerated and associated with substantial and persistent improvements in functional state, exercise capacity, and pulmonary haemodynamics.

Beraprost sodium is a prostacyclin analogue that is suitable for oral administration owing to its stable structure (cyclo-pentabenzofuranyl skeleton). Its half life is 45 minutes in the fasting state and it lasts 3-3.5 hours when taken after a meal. Like other prostacyclin analogues, beraprost produces vasodilatation, inhibition of platelet aggregation, and inhibition of smooth muscle cell proliferation. ${ }^{22}$ Acute administration of beraprost in patients with pulmonary hypertension has been shown to cause pulmonary vasodilatation when used alon $e^{13}$ or in combination with inhaled nitric oxide. ${ }^{23}$ Two recent retrospective studies suggest that oral beraprost added to conventional treatment induces a long lasting haemodynamic improvement together with a reduction in mortality compared with a historical control group treated conventionally. ${ }^{14} 15$

There have until now been few randomised controlled trials of intravenous epoprostenol (prostacyclin) in primary pulmonary hypertension $^{23}$ or pulmonary hypertension secondary to connective tissue disease. ${ }^{7}$ In primary pulmonary hypertension, three months of intravenous epoprostenol increased the six minute walk distance by an average of about $50 \mathrm{~m}$, compared with a decrease by an average of $70 \mathrm{~m}$ in controls. ${ }^{2}$ In that study, there were eight deaths in the control group, and no mortality in the treatment group. ${ }^{2}$ In pulmonary hypertension secondary to connective tissue disease, three months of intravenous epoprostenol increased the six minute walk distance by an average of $46 \mathrm{~m}$, compared with an average decrease of $48 \mathrm{~m}$ in the controls. ${ }^{7}$ In both studies, epoprostenol decreased mean pulmonary artery pressure only moderately, on average by no more than $5 \mathrm{~mm} \mathrm{Hg}$, but cardiac output increased greatly, so that pulmonary vascular resistance decreased on average by 3.4 to 4.6 Wood units, or around $25 \%$ from baseline. ${ }^{2}$ 
Open uncontrolled series of patients with primary or secondary hypertension treated with intravenous epoprostenol for one year or more show more important improvements in pulmonary haemodynamics and exercise capacity, with a decrease in pulmonary vascular resistance within a range of $35-50 \%$, and an increase in the six minute walk distance within a range of $60-100 \mathrm{~m}$ (or an equivalent improvement in exercise tests). ${ }^{49}$

Nebulised iloprost has been reported in an uncontrolled study to improve exercise capacity and pulmonary haemodynamics after three months of treatment in patients with pulmonary hypertension, either primary or secondary to connective tissue disease or peripheral thromboembolism. ${ }^{11}$ A recent uncontrolled study showed that patients with primary pulmonary hypertension treated with nebulised iloprost for one year had on average an increase in their six minute walk distance of 85 metres and a decrease in pulmonary vascular resistance by $23 \% .^{12}$

In our patients treated for one year with oral beraprost, the six minute walk distance increased by an average of $63 \mathrm{~m}$, which is comparable to the reported improvements after one year of intravenous epoprostenol or inhaled iloprost. The decrease in systolic pulmonary artery pressure was on average only $8 \mathrm{~mm} \mathrm{Hg}$ and of borderline significance. However, it is difficult to translate this measurement into pulmonary vascular resistance, as mean pulmonary artery pressure and cardiac output were not measured. In view of associated changes in functional state and exercise capacity, it may be that the beraprost induced decrease in pulmonary vascular resistance was of the same magnitude as reported after nebulised iloprost or intravenous epoprostenol. Such effects are likely to be associated with improved long term survival, as reported with intravenous epoprostenol ${ }^{45}$ and oral beraprost. ${ }^{15}$

The dose of beraprost in our patients was determined on the basis of limited previous experience in patients with pulmonary hypertension, and titrated by clinical improvement and side effects. After one year, our patients were receiving $100-360 \mu \mathrm{g} / \mathrm{day}$, which is more than the dose of 60-180 $\mu \mathrm{g}$ split in three to four doses a day reported to improve survival in patients with primary pulmonary hypertension in a retrospective study with historical controls. ${ }^{15}$ The optimal dose of beraprost is not yet known precisely, so it appears reasonable to increase the dose progressively until limited by side effects in patients with a good clinical response.

The side effects in our patients were similar to those of the prostacyclins in general-that is, skin flushing, rash, arthralgia or muscle pain, and gastrointestinal discomfort with nausea or diarrhoea. ${ }^{18}$ Side effects less specific to prostacyclin have been reported in patients treated with nebulised iloprost. ${ }^{11}{ }^{12}$ Intravenous epoprostenol carries the life threatening risks of catheter related thrombosis, sepsis, and pneumothorax, and also exposes the patients to delivery system failures leading to rapid overdosing or underdosing. ${ }^{18}$ On the other hand, nebulised iloprost may be associated with coughing, and there is limitation of mobility and quality of life because of the time consuming and frequent inhalations. ${ }^{11}{ }^{12}$ Both intravenous epoprostenol and inhaled iloprost are expensive treatments, with annual costs estimated for Europe to range from 50000 to 300000 Euros or more, not including expenses related to delivery devices and disposal. ${ }^{12}$

\section{LIMITATIONS}

The obvious limitations of our study are the small number of patients and the absence of controls. It must also be remembered that our patients, at the time of their inclusion, could not benefit from the only treatment shown by a randomised controlled trial to decrease mortality in severe pulmonary hypertension, intravenous epoprostenol. Thus beraprost was prescribed on a compassionate basis, with no alternative available in the presence of ineffective conventional treatment. However, it is pertinent to ask whether the clinical benefit observed after one year in 11 of our 13 patients might have occurred by chance. We believe this is very unlikely, in view of the known natural history of the disease as established on large series of patients, ${ }^{16}$ and confirmed in randomised controlled studies extending over three month periods of observation. ${ }^{237}$ In particular, over a year of follow up pulmonary hypertension of the severity seen in our patients would be expected to be associated with a decline in the six minute walk distance and a deterioration, instead of an improvement, in functional class, if not one or two deaths. ${ }^{2} 3716$

\section{CONCLUSIONS}

A randomised controlled study of oral beraprost may be warranted in patients with severe pulmonary hypertension who do not improve with optimal conventional treatment.

1 Higenbottam TW, Spiegelhalter D, Scott JP, et al. Prostacyclin (epoprostenol) and heart-lung transplantation as treatments for severe pulmonary hypertension. Br Heart $f$ 1993;70:366-70.

2 Rubin LJ, Mendoza J, Hood M, et al. Treatment of primary pulmonary hypertension with continuous intravenous prostacyclin (epoprostenol). Results of a randomized trial. Ann Intern Med 1990;112:485-91.

3 Barst RJ, Rubin LJ, McGoon MD, et al. Survival in primary pulmonary hypertension with long-term continuous intravenous prostacyclin. Ann Intern Med 1994;121:409-15.

4 Barst RJ, Rubin LJ, Long WA, et al. A comparison of continuous intravenous epoprostenol (prostacyclin) with conventional therapy for primary pulmonary hypertension. N Engl f Med 1996;334:296-301.

5 Shapiro SM, Oudiz RJ, Cao T, et al. Primary pulmonary hypertension: improved long-term effects and survival with continuous intravenous epoprostenol infusion. $\mathcal{F}$ Am Coll

6 McLaughlin VV, Genthner DE, Panella MM, et al. Reduction in pulmonary vascular resistance with long-term epoprostenol (prostacyclin) therapy in primary pulmonary hypertension. N Engl f Med 1998;338:273-7.

7 Badesch DB, Tapson VF, McGoon MD, et al. Continuous intravenous epoprostenol for pulmonary hypertension due to scleroderma spectrum disease. A randomized controlled trial. Ann Intern Med 2000;132:425-34.

8 Humbert M, Sanchez O, Fartoukh M, et al. Short-term and long-term epoprostenol (prostacyclin) therapy in pulmonary hypertension secondary to connective tissue diseases: results of a pilot study. Eur Respir F 1999;13:1351-6.

9 Rosenzweig, Kerstein D, Barst RJ. Long-term prostacyclin for pulmonary hypertension with associated congenital heart defects. Circulation 1999;99:1858-65.

10 McLaughlin VV, Genthner DE, Panella MM, et al. Compassionate use of continuous prostacyclin in the management of secondary pulmonary hypertension: a case series. Ann Intern Med 1999;130:740-3. 
11 Olschewski H, Ghofrani HA, Schmehl T, et al. Inhaled iloprost to treat severe pulmonary hypertension. An uncontrolled trial. German PPH Study Group. Ann Intern Med 2000; 132:435-43.

12 Hoeper MM, Schwarze M, Ehlerding S, et al. Long-term treatment of primary pulmonary hypertension with aerosolized iloprost, a prostacyclin analogue. $N$ Engl f $\mathrm{Med}$ 2000;342:1866-70

13 Saji T, Ozawa Y, Ishikita T, et al. Short-term hemodynamic effect of a new oral $\mathrm{PGI}_{2}$ analogue, beraprost, in primary and secondary pulmonary hypertension. Am $\mathcal{f}$ Cardiol 1996;78:244-7.

14 Okano Y, Yoshioka T, Shimouchi A, et al. Orally active prostacyclin analogue in primary pulmonary hypertension. Lancet 1997;349:1365.

15 Nagaya N, Uematsu M, Okano Y, et al. Effect of orally active prostacyclin analogue on survival of outpatients with primary pulmonary hypertension. $\mathcal{F}$ Am Coll Cardiol 1999; 34:1188-92.

16 Rich S, Dantzker DR, Ayres SM, et al. Primary pulmonary hypertension: a national prospective study. Ann Intern Med. 1987;107:216-23.
17 Moser KM, Auger WR, Fedullo PF, et al. Chronic thromboemeric pulmonary hypertension: clinical picture and surembolic pulmonary hypertension: clinical pictu
gical treatment. Eur Respir f 1992;5:334-42.

18 Rubin LJ. Primary pulmonary hypertension. N Engl f Med 1997;336:111-17

19 Guyatt GH, Thompson PJ, Berman LB, et al. How should we measure function in patients with chronic heart and lung disease? f Chronic Dis 1985;38:517-24.

20 Yock PG, Popp RL. Noninvasive estimation of right ventricular systolic pressure by Doppler ultrasound in patients with tricuspid regurgitation. Circulation 1984;70: 667-71.

21 Winer BJ. Statistical principles in experimental design. New York: McGraw-Hill, 1971.

22 Murata T, Murai T, Kanai T, et al. General pharmacology of beraprost sodium, second communication: effect on the autonomic, cardiovascular and gastrointestinal on the autonomic, cardiovascular and gastrointestinal systems,

23 Ichida $\mathrm{F}$, Uese $\mathrm{K}$, Trubata $\mathrm{S}$, et at. Additive effect of beraprost on pulm Ker rost on pulmonary vasodilation by inhaled nitric oxide in 80:662-4.

\section{IMAGES IN CARDIOLOGY}

\section{A case of cardiac angiosarcoma presenting as pericardial tamponade}

A 67 year old woman presented with syncope. Physical examination revealed pulsus paradoxus $20 \mathrm{~mm} \mathrm{Hg}$ and jugular venous distention, leading to a diagnosis of pericardial effusion and possible tamponade. Echocardiogram showed massive circumferential pericardial effusion, diastolic right ventricular collapse, and dilated inferior vena cava without respiratory changes. Pericardiocentesis yielded $1100 \mathrm{ml}$ of serosanguinous fluid. All cytological and bacteriological tests on the fluid were negative. Repeat echocardiogram showed significant pericardial effusion posteriorly. Chest computed tomography (CT) scan showed large bilateral pleural effusion and a $3 \mathrm{~cm}$ mass at the junction of the right atrium (RA) and the right ventricle (RV), probably blood or tumour (top right).

Transoesophageal echocardiography showed a mass in the pericardial sac between the right atrium and the right ventricle extending off the atrioventricular groove and into the right atrium (bottom right).

Surgical exploration revealed an extremely vascular pericardium with dense adhesion to the epicardium. A mass was palpated in the right atrium and the wall of the right ventricle. Wedge biopsy showed this was angiosarcoma. The patient received chemotherapy with doxorubicin and is currently in remission.

Most cardiac tumours are secondary; $75 \%$ of the primary tumours are benign, and $75 \%$ of the malignant tumours are sarcomas. Widespread metastases, especially pulmonary, are common by the time the diagnosis is made. Since the signs and symptoms are non-specific, the diagnosis is often delayed which leads to
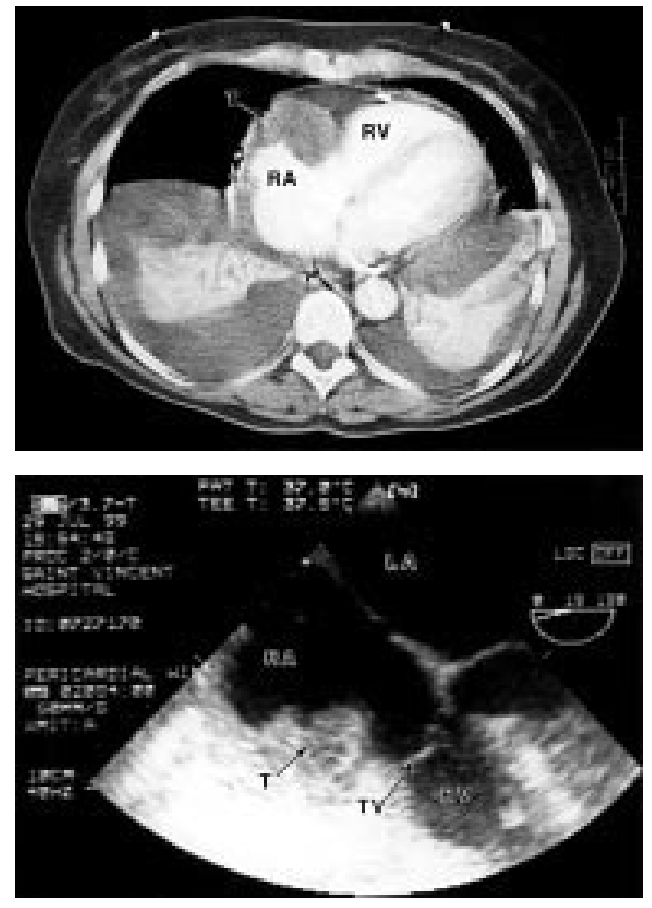

poor outcomes. Diagnostic imaging such as CT scan, transoesophageal echocardiography, and magnetic resonance imaging may allow early diagnosis and treatment. Several combinations of treatment strategies were tried, but the optimum therapy is still unknown.

HUSAM H FARAH MINA JACOB JAYASHRI ARAGAM david.spodick@tenethealth.com 